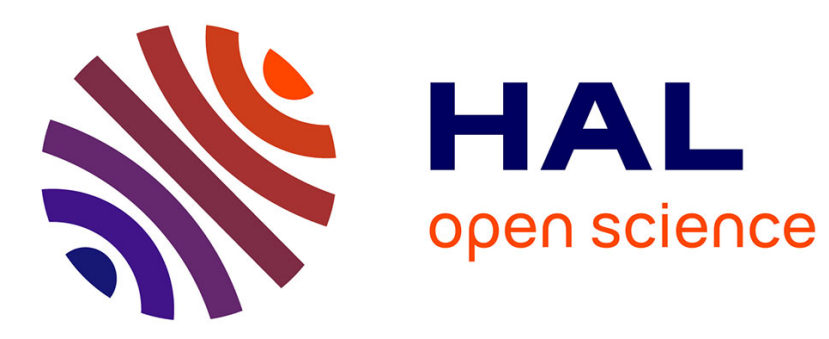

\title{
Semianalytical solution for the stress distribution in notched tubes
}

\author{
Mohamed Ferjani, Daniel Averbuch, Andrei Constantinescu
}

\section{To cite this version:}

Mohamed Ferjani, Daniel Averbuch, Andrei Constantinescu. Semianalytical solution for the stress distribution in notched tubes. International Journal of Fatigue, 2011, 33, pp. 557-567. 10.1016/j.ijfatigue.2010.10.009 . hal-00544303

\section{HAL Id: hal-00544303}

https://hal-polytechnique.archives-ouvertes.fr/hal-00544303

Submitted on 8 Dec 2010

HAL is a multi-disciplinary open access archive for the deposit and dissemination of scientific research documents, whether they are published or not. The documents may come from teaching and research institutions in France or abroad, or from public or private research centers.
L'archive ouverte pluridisciplinaire HAL, est destinée au dépôt et à la diffusion de documents scientifiques de niveau recherche, publiés ou non, émanant des établissements d'enseignement et de recherche français ou étrangers, des laboratoires publics ou privés. 


\title{
Semianalytical solution for the stress distribution in notched tubes
}

\author{
Mohamed Ferjani ${ }^{\mathrm{a}, \mathrm{b}}$ Daniel Averbuch ${ }^{\mathrm{b}}$ Andreï Constantinescu ${ }^{\mathrm{a}}$ \\ ${ }^{a}$ Solid Mechanics Laboratory - CNRS UMR 7649, \\ Department of Mechanics - Ecole Polytechnique, \\ 91128 Palaiseau cedex, France \\ ${ }^{\mathrm{b}}$ IFP Energies nouvelles, \\ Rond-point de l'Ál'changeur de Solaize, \\ BP 3, 69360 Solaize, France \\ Corresponding author : Andreï Constantinescu \\ andrei.constantinescu@lms.polytechnique.fr, \\ tel : (33) 169335756 fax : (33) 169333026
}

\begin{abstract}
The aim of this paper is to present an alternative solution to the finite element method for the determination of the elastic stress field for an axi-symmetric notched tube under tension and bending loadings. The proposed solution is an extension of the work of Filippi and Lazzarin (2004) to tubular specimens. It provides the stress field distribution along the notch bisector. A good agreement was found between the solution proposed in the present paper and the results from a finite element analysis. A sensitivity analysis is performed to establish the domain of validity of the solution with respect to the dimensions of the notched tube
\end{abstract}

Key words:

Notch, closed-form solution, stress concentration factor, tube.

\section{List of symbols}

Preprint submitted to International Journal of Fatigue 


$\begin{array}{ll}\underline{\underline{\sigma}} & \text { stress field associated to the local system of coordinates } \\ \underline{\underline{\Sigma}} & \text { stress field associated to the global system of coordinates } \\ \rho & \text { root radius of the notch } \\ \alpha & \text { half opening angle of the notch } \\ R_{e}, R_{i} \text { and } R_{O} & \text { external, internal and notch tip radii of the tube } \\ p & \text { depth of the notch } \\ l & \text { ligament length of the notch } \\ e & \text { thickness of the tube } \\ K_{Z Z}^{t} & \text { axial stress concentration factor for tension loading } \\ K_{Z Z}^{f} & \text { axial stress concentration factor for bending loading } \\ \xi & \text { dimensionless parameter characterizing the geometry } \\ & \text { of the notched tube }\end{array}$

\section{Introduction}

Fatigue design is a complex engineering field where materials, geometry and loading define the lifetime of a structure. The fatigue design methodology is a two step process based on a mechanical analysis devoted to the computation of the stresses and a fatigue analysis devoted to lifetime prediction. As fatigue cracks initiate favorably near notches, holes or generally in regions of stress concentration, it is important to estimate stresses precisely in these parts in order to obtain accurate lifetime predictions.

Additional difficulties to estimate the fatigue life of notched components come from different factors which are essentially related to the stress gradient $[29,25,12,13]$ or the scale effect $[10,26,20]$. These factors influence the fatigue process leading to unsatisfactory fatigue predictions when classical fatigue criteria such as the Crossland criterion or the Findley criterion are employed.

Fortunately the application of these fatigue criteria combined with different methods such as the critical distance $[15,23,30]$ or modified to incorporate the influence of the stress gradient [24] shows good agreements to estimate fatigue limits. However, all these techniques need the knowledge of the stress distribution near the notch tip either to compute the stress gradient or to compute the stress components at a different distance from notch tip. 
To achive the first step of the fatigue design methodology, engineers can use different numerical or analytical methods and tools. Numerical methods like finite boundary elements method provide solutions for a large class of geometries, but are mesh dependent in areas of stress concentration and time consuming. Moreover, sensitivity analysis with respect to different parameters will further increase the computational burden.

Classical closed-form solutions for infinite bodies containing holes have been presented by Savin [28] based on the complex potentials proposed previously by Muskeshishvilli [21]. This solution employs the theory of complex elasticity which has been a powerful mathematical tool for many different problems such as contact problem [14], slope stability [18] or crack problem [3].

Several papers $[31,17,11,4]$ have proposed expressions for the stress field ahead of a notch depending on two parameters: the notch root radius $\rho$ and the axial stress concentration factor $K_{t}$. Indeed, they showed that in the vicinity of the notch tip, the stress field distribution is more influenced by the notch root radius than by the global geometry of the notch and is very similar for a variety of notches when stresses are normalized by the stresses at the notch tip.

Thus the stress distribution obtained by these solutions, when considered away from the notch, is not dependent on the stress concentration factor, which is incoherent with the assumptions of the analysis [27]. Therefore these solutions are not accurate enough [5] for a distance ahead of the notch tip larger than $3 \rho$ in the case of a sharp notch $\left(K_{t}>5\right)$ and $\rho$ in the case of blunt notch $\left(K_{t}<5\right)$.

$[16,9,2]$ proposed elaborate approximate closed-form expressions for a unique solution applicable both for blunt and sharp notches. Their solutions are valid for notched bars and plates under tension and bending loading.

The present work proposes a further extension of these solutions in the form of an approximate stress field solution devoted to tubes presenting an internal or an external notch under tension and bending loadings. The application of this solution to a threaded connection is presented in $[6,7]$

The paper is organized as follows: the next section presents a short review of the solution proposed by Filippi and Lazzarin (2004) dedicated to notched bars. The third section is dedicated to the extension of the preceding solution to tubular geometries with an external or an internal notch and the determination of the different parameters introduced in the solution. The final section discusses the comparisons of the semi-analytical solution with the finite element results for a variety of notch geometries and defines the validity of the solution with regards to a dimensionless parameter $\xi$ representing a ratio between notch depth and the tube thickness. 


\section{Stress problem for notched tubes}

In this section, we are interested in the formulation of an approximate solution of the elastic stress field $\underline{\underline{\underline{\Sigma}}}$ in the vicinity of a notched axi-symmetrically tube under tension and bending loading.

The geometry of the tubes is depicted in Fig. 1. We shall consider two systems of coordinates, the former is a global system of coordinates $\left\{\underline{e}_{R}, \underline{e}_{\Theta}, \underline{e}_{Z}\right\}$ with the $\mathrm{z}$-axis along the axis of the tube and the latter is a local system of coordinates $\left\{\underline{e}_{x}, \underline{e}_{y}, \underline{e}_{z}\right\}$ in the neighborhood of the notch. The tube is defined by an internal and an external radius, $R_{i}$ and $R_{e}$ respectively. The notch has its tip at a distance $R_{o}$ from the axis of the tube and its depth $p$ is equal to

$$
p=\left\{\begin{array}{c}
R_{e}-R_{o} \text { for external notch } \\
R_{o}-R_{i} \text { for internal notch }
\end{array}\right.
$$

The notch has a rounded V-shaped geometry with an opening angle $2 \alpha$ and a root radius $\rho$.

Along the bisector of the notch, the stress field $\Sigma$ has the following matrix form for tension and bending loading in the global system of coordinates $\left\{\underline{e}_{R}, \underline{e}_{\Theta}, \underline{e}_{Z}\right\}$, due to the symmetry of the problem, and to some simplifications regarding the order of magnitude of the stress components with respect to the axial component $\Sigma_{Z Z}$

$$
\underline{\underline{\underline{\Sigma}}}=\left[\begin{array}{ccc}
\Sigma_{R R} & 0 & 0 \\
0 & \Sigma_{\Theta \Theta} & 0 \\
0 & 0 & \Sigma_{Z Z}
\end{array}\right]
$$

On the same bisector, we shall denote $\underline{\underline{\sigma}}$ the associated stress field expressed in the local system of coordinates $\left\{\underline{e}_{x}, \underline{e}_{y}, \underline{e}_{z}\right\}$. In our case, its components are defined by

$$
\underline{\underline{\sigma}}=\left[\begin{array}{ccc}
\sigma_{x} & 0 & 0 \\
0 & \sigma_{y} & 0 \\
0 & 0 & \sigma_{z}
\end{array}\right]=\left[\begin{array}{ccc}
\Sigma_{R R} & 0 & 0 \\
0 & \Sigma_{Z Z} & 0 \\
0 & 0 & \Sigma_{\Theta \Theta}
\end{array}\right]
$$

One can note that the shear component $\sigma_{x y}$ is equal to zero everywhere along the notch ligament, i.e $0>r-r_{o}>R_{e}-R_{o}$, due to the symmetry of the problem. 
Recently, a set of closed-form equations valid for V-shaped notched axi-symmetric bars, i.e $R_{i}=0$, subjected to mode I loadings (tension and bending) has been proposed in [8].

The local elastic stress field $\underline{\underline{\sigma}}$ has been obtained with the use of the KolosoffMushkelishvili's complex potential function [21] and the conformal mapping introduced by Neuber [22]. This solution has been obtained under plane stress or plane strain condition with respect to the local coordinate system, which is verified for sufficiently large $R$.

The solution in [8] for the stress field $\underline{\underline{\sigma}}$ along the notch bisector, i.e. $y=0$ or $\theta=0$, of a cylindrical bar expressed in the local system of coordinates $\left\{\underline{e}_{x}, \underline{e}_{y}, \underline{e}_{z}\right\}$ (see Fig. 1) is

$$
\begin{aligned}
\sigma_{y} & =\frac{\sigma_{\max }}{4(q-1)+q \omega_{1}} g(r)\left[4(q-1) f(r)^{\lambda_{1}-1}+q \omega_{1}\left(\frac{r}{r_{o}}\right)^{\mu_{1}-1}\right], \\
\sigma_{x} & =\frac{\sigma_{\max }}{\eta_{1}\left[4(q-1)+q \omega_{1}\right]}\left[4(q-1)\left(\frac{r}{r_{o}}\right)^{\lambda_{1}-1}+q \delta_{1}\left(\frac{r}{r_{o}}\right)^{\mu_{1}-1}\right]
\end{aligned}
$$

where $\sigma_{\max }$ is the maximum stress in the $y$-direction and functions $f(r)$ and $g(r)$ will be defined later. The other parameters $q, r_{o}, \lambda_{1}, \mu_{1}, \omega_{1}, \delta_{1}$ and $\eta_{1}$ involved in (4) and (5) are defined in Appendix 1.

The stress field defined by (4) and (5) is an approximate solution, in the sense that the free surface boundary condition is satisfied only at the notch tip and on the rectilinear flanks of the V-notch, where the boundary conditions were set according to Williams [19].

Originally [8] the function $f(r)$ in (4) was linear, expressed as: $\frac{r}{r_{o}}$. This solution gives good results close to the neighborhood of the notch tip but becomes less accurate far away from it. Thereby, in order to obtain a better solution all along the notch bisector, the function $f(r)$ was modified in the form

$$
f(r)=1+\frac{\operatorname{atan}\left[m\left(r-r_{o}\right)\right]}{m r_{o}}
$$

where the parameter $m$ will be defined later. This solution has shown good agreements with respect to FEM results [8] . It is worth noting that (6) matches the linear law $\frac{r}{r_{o}}$ in the vicinity of the notch tip.

The function $g(r)$ expressed in (4) is related to the loading mode, tension or bending, and is equal to 


$$
g(r)= \begin{cases}1-\frac{r-r_{o}}{\kappa} & \text { for bending } \\ 1 & \text { for tension }\end{cases}
$$

where $\kappa$ is the distance from the neutral axis to the notch tip, and is equal to $R_{o}$.

At this stage, the solution is complete up to the determination of the parameter $m$ (see [8]) and to the determination of $\sigma_{\max }$ :

In order to identify the best values of these two parameters, for a given geometry, two additional relations are then needed. These two values will then be identified by equating the theoretical axial stress concentration factor $K_{Z Z}$, with the stress concentration $K_{t}$ computed with the finite element method, and by writing the global equilibrium condition of the tube. As it will be shown later in section 5., the value of the parameter $m$ varies only weakly with the notch root radius. This result, equally reported in [8], permits to perform the sensitivity studies without performing additional finite element analysis, and justifying the pratical interest in the present approach.

The results can be summerized by the following expressions:

$$
\begin{aligned}
& \Sigma_{R R}=\frac{E}{1-\nu^{2}}\left(\frac{d u_{R}}{d R}+\nu \frac{u_{R}}{R}\right)+\Sigma_{Z Z} \frac{\nu}{1-\nu}, \\
& \Sigma_{\Theta \Theta}=\frac{E}{1-\nu^{2}}\left(\frac{u_{R}}{R}+\nu \frac{d u_{R}}{d R}\right)+\Sigma_{Z Z} \frac{\nu}{1-\nu}
\end{aligned}
$$

where $E$ and $\nu$ are the Young's modulus and the Poisson's ratio, respectively, whereas $u_{R}$ is the radial displacement. The first of the previous equations provides the radial displacement by integration with an initial condition $u_{R}=0$ at $R=0$ which is valid for bars only. Then the distribution of the hoop stress, with respect to $R$, is obtained by substituting the solution $u_{R}$ into the second equation.

\section{Local formulation of the solution for notched tubes}

In order to extend this solution to tubular geometries subjected to tension and bending loadings, the stress component $\sigma_{x}$ must satisfy the free surface condition, i.e. $\sigma_{x}=0$ at $R=R_{i}$ and $R=R_{e}$ for an internal and external notched tube, respectively.

As a consequence the function $h(r)$ is added to (5) such that the boundary condition is satisfied. Thus, the stress component $\sigma_{x}$ becomes 


$$
\sigma_{x}=\frac{\sigma_{\max }}{\eta_{1}\left[4(q-1)+q \omega_{1}\right]}\left[4(q-1)\left(\frac{r}{r_{o}}\right)^{\lambda_{1}-1}+q \delta_{1}\left(\frac{r}{r_{o}}\right)^{\mu_{1}-1}-h(r) \Gamma\right]
$$

where the function $h(r)$ satisfies the two following conditions

$$
h\left(r_{o}\right)=0 \quad \text { and } \quad h\left(r_{o}+l\right)=1 .
$$

Moreover, the coefficient $\Gamma$ in (10) and the ligament length $l$ in (11) are given by

$$
\begin{aligned}
& \Gamma=4(q-1)\left(1+\frac{l}{r_{o}}\right)^{\lambda_{1}-1}+q \delta_{1}\left(1+\frac{l}{r_{o}}\right)^{\mu_{1}-1}, \\
& l= \begin{cases}R_{o}-R_{i} & \text { ext } \\
R_{e}-R_{o} & \text { int }\end{cases}
\end{aligned}
$$

Here 'ext' and 'int' simply denote the formula to use for an external or an internal notch, respectively.

The simplest function satisfying (11) is the linear function, thus

$$
h(r)=\frac{r-r_{o}}{l}
$$

For the component $\sigma_{y}$, its expression is given by

$$
\sigma_{y}=\frac{\sigma_{\max }}{4(q-1)+q \omega_{1}} g(r)\left[4(q-1) f(r)^{\lambda_{1}-1}+q \omega_{1}\left(\frac{r}{r_{o}}\right)^{\mu_{1}-1}\right]
$$

with the modified $g$ function

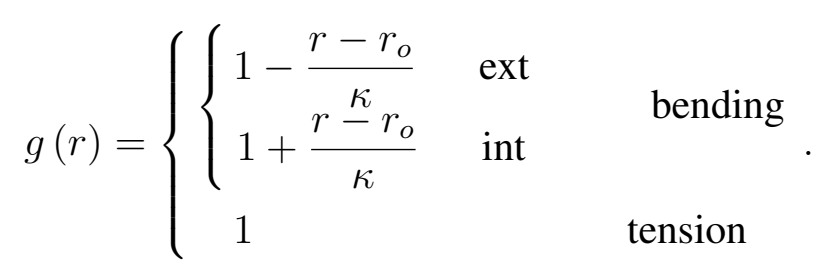

Therefore the axial $\Sigma_{Z Z}$ and the radial $\Sigma_{R R}$ components of the stress field $\underline{\underline{\Sigma}}$ along the ligament are equal to 


$$
\begin{aligned}
& \Sigma_{Z Z}=\frac{\sigma_{\max }}{4(q-1)+q \omega_{1}} g(r)\left[4(q-1) f(r)^{\lambda_{1}-1}+q \omega_{1}\left(\frac{r}{r_{o}}\right)^{\mu_{1}-1}\right], \\
& \Sigma_{R R}=\frac{\sigma_{\max }}{\eta_{1}\left[4(q-1)+q \omega_{1}\right]}\left[4(q-1)\left(\frac{r}{r_{o}}\right)^{\lambda_{1}-1}+q \delta_{1}\left(\frac{r}{r_{o}}\right)^{\mu_{1}-1}\right. \\
& -h(r) \Gamma] .
\end{aligned}
$$

Once again, the hoop stress can be determined with the help of the constitutive equations 9. One can however remark that the initial condition used for bars : $u_{R}=$ 0 for $R=0$, has to be modified. For this reason, we assume that, far away from the notch tip, the mechanical fields are the same as for a tube without the notch. In other words, we assume that $\Sigma_{\Theta \Theta}=0$ at $R=R_{i}$ for the case of an external notch and $\Sigma_{\Theta \Theta}=0$ at $R=R_{e}$ for the case of an internal notch. Solving for $u_{R}$ in (8) and (9) and using the boundary condition, one obtains:

$$
\begin{array}{ll}
\left.u_{R}\right|_{R=R_{i}}=-\left.\frac{\nu R_{i}}{E} \Sigma_{Z Z}\right|_{R=R_{i}} & \text { external } \\
\left.u_{R}\right|_{R=R_{e}}=-\left.\frac{\nu R_{e}}{E} \Sigma_{Z Z}\right|_{R=R_{e}} & \text { internal }
\end{array}
$$

Finally the hoop stress $\Sigma_{\Theta \Theta}$ is determined by introducing $u_{R}$ and $\Sigma_{Z Z}$ into (9).

\section{Global formulation of the solution for notched tubes}

At this stage, the solution is complete up to the determination of the parameter $m$ (see (6)) which belongs to the open range $[0,+\infty[$ and depends on the notch geometry and the relative dimensions of the tubular components. This parameter is obtained numerically by equaling the theoretical axial stress concentration factor $K_{Z Z}$ with the stress concentration $K_{t}$ computed with the finite element method

$$
K_{t}=K_{Z Z}=\frac{\sigma_{\max }}{\sigma_{\text {nom }}}
$$

where $\sigma_{\text {nom }}$ is the applied gross nominal stress and $\sigma_{\max }$ is the maximal axial stress as depicted in Fig. 2. It is well known, that the precision of the numerical computed stress concentration is influenced by the mesh density. A short discussion of this point will be presented in the next section and is illustred in Fig. 4. 
Invoking the global equilibrium condition for tension and bending loadings, one can obtain an equation which can be solved numerically as a function of the parameter $m$.

The global equilibrium condition for tension loading takes the following form

$$
\begin{aligned}
& T=\left\{\begin{array}{lc}
\int_{R i}^{R_{o}} \Sigma_{Z Z}(R) 2 \pi R d R & \text { ext } \\
\int_{R_{o}}^{R_{e}} \Sigma_{Z Z}(R) 2 \pi R d R & \text { int }
\end{array}\right. \\
& R= \begin{cases}R_{o}-r+r_{o} & \text { ext } \\
R_{o}+r-r_{o} & \text { int }\end{cases}
\end{aligned}
$$

where $T$ is the tension load linked to the gross nominal stress $\sigma_{\text {nom }}$ by

$$
T=\pi\left(R_{e}^{2}-R_{i}^{2}\right) \sigma_{\text {nom }}
$$

Substituting (17) into (22) and rearranging the obtained equation, one obtains the following relation for $K_{Z Z}^{t}$ for tension loading

$$
K_{Z Z}^{t}=\frac{\left(R_{e}^{2}-R_{i}^{2}\right)\left[4(q-1)+q \omega_{1}\right]}{2\left(A_{m}+B\right)}
$$

with

$$
\begin{aligned}
A_{m}=4(q-1) \int_{0}^{l}\left\{1+\frac{\operatorname{atan}(m x)}{m r_{o}}\right\}^{\lambda_{1}-1}\left(R_{o} \pm x\right) d x \\
B=\frac{q \omega_{1} r_{o}}{\mu_{1}}\left\{R_{o}\left[\left(1+\frac{l}{r_{o}}\right)^{\mu_{1}}-1\right]\right. \\
\pm\left[\frac{r_{o}}{\mu_{1}+1}\left(1+\frac{l}{r_{o}}\right)^{\mu_{1}+1}-\frac{l}{r_{o}}\left[\left(1+\frac{l}{r_{o}}\right)^{\mu_{1}}-1\right]\right]
\end{aligned}
$$

where the signs + and - in (26) and (27) stand for an internal and external notch, respectively.

For bending loading (see Fig. 3), the balance of angular moment is employed after having specified which is the peak stress $\sigma_{\max }$ to use in (8) and which is the distance $\kappa$ to introduce in (7). To carry out the calculation, we assume that along a vertical 
strip $d X$, as depicted in Fig. 3, the axial stress component $\Sigma_{Z Z}$ is given by (17) and the peak stress $\sigma_{\max }$ is only a function of $X$ such that

$$
\sigma_{\max }(X)=\sigma_{\max }^{\prime} \sqrt{1-\left(\frac{X}{R_{o}}\right)^{2}}
$$

where $\sigma_{\max }^{\prime}$ is the maximum axial stress at $X=0$.

Regarding the strip $d X$, the parameter $\kappa$ is now defined as the distance between the notch tip at $X$ and the plan $Y=0$ and, as for the stress peak, we assume that $\kappa$ is equal to

$$
\kappa=R_{o} \sqrt{1-\left(\frac{X}{R_{o}}\right)^{2}} .
$$

Moreover, in the case of an internal notch, the stress component $\Sigma_{Z Z}$ is not defined in the region $R_{e} \geq X \geq R_{o}$, therefore we assume that, in this region, $\Sigma_{Z Z}$ is equal to the axial stress of the same geometrical tube without notch, thus

$$
\Sigma_{Z Z}^{*}=\frac{Y}{\kappa} \sigma_{n o m}
$$

Therefore, under the preceeding assumptions, the balance of angular moment becomes

$$
\begin{aligned}
& \frac{M}{4}=\left\{\begin{array}{l}
\int_{0}^{R_{i}} \int_{\sqrt{R_{i}^{2}-X^{2}}}^{\sqrt{R^{2}-X^{2}}} \Sigma_{Z Z} Y d Y d X+\int_{R_{i}}^{R_{o}} \int_{0}^{\sqrt{R_{o}^{2}-X^{2}}} \Sigma_{Z Z} Y d Y d X \\
\text { for an external notch } \\
\int_{0}^{R_{o}} \int_{\sqrt{R_{o}^{2}-X^{2}}}^{\sqrt{R^{2}}} \Sigma_{Z Z} Y d Y d X+\int_{R_{o}}^{R_{e}} \int_{0}^{\sqrt{R_{e}^{2}-X^{2}}} \Sigma_{Z Z}^{*} Y d Y d X
\end{array}\right. \\
& Y=\left\{\begin{array}{ll}
\sqrt{R_{o}^{2}-X^{2}}+r_{o}-r & \text { for an internal an external } \\
\sqrt{R_{o}^{2}-X^{2}}-r_{o}+r & \text { for an internal }
\end{array} \quad \text { and for } \quad X \leq R_{o}(32)\right.
\end{aligned}
$$

where $M$ denotes the bending load that related to the maximal gross nominal stress $\sigma_{\text {nom }}$ by 


$$
\begin{aligned}
& M=\frac{I}{R_{e}} \sigma_{\text {nom }}, \\
& I=\frac{\pi}{4}\left(R_{e}^{4}-R_{i}^{4}\right) .
\end{aligned}
$$

Substituting (17) into (31), one obtains for bending loading the expression for the axial stress concentration factor $K_{Z Z}^{f}$

$$
K_{Z Z}^{f}=R_{o}\left(\frac{I}{4 R_{e}}-G\right) \frac{4(q-1)+q \omega_{1}}{C_{m}}
$$

with

$$
\begin{aligned}
& C_{m}=\left\{\begin{array}{cc}
\left\{\int_{0}^{R_{i}} \int_{\sqrt{R_{i}^{2}-X^{2}}}^{\sqrt{R_{2}^{2}}}+\int_{R_{i}}^{R_{o}} \int_{0}^{\sqrt{R_{o}^{2}-X^{2}}} \cdot\right\} \mathcal{G}_{m}(Y, X) Y^{2} d Y d X & \text { ext } \\
\int_{0}^{R_{o}} \int_{\sqrt{R_{e}^{2}-X^{2}}}^{\sqrt{R^{2}}} \mathcal{G}_{m}(Y, X) Y^{2} d Y d X & \text { int }
\end{array}\right. \\
& G=\left\{\begin{array}{c}
0 \\
\int_{R_{o}}^{R_{e}} \int_{0}^{\sqrt{R_{e}^{2}-X^{2}}} \Sigma_{Z Z}^{*} Y d Y d X \quad \text { int }
\end{array}\right. \\
& \mathcal{G}_{m}(Y, X)=4(q-1)\left\{1 \pm \frac{\operatorname{atan}\left[m\left(Y-\sqrt{R_{o}^{2}-X^{2}}\right)\right]}{m r_{o}}\right\}^{\lambda_{1}-1} \\
& +q \omega_{1}\left(1 \pm \frac{Y-\sqrt{R_{o}^{2}-X^{2}}}{r_{o}}\right)^{\mu_{1}-1}
\end{aligned}
$$

where the signs + and - in (38) stand respectively for an internal and external notch.

\section{Results and limitations of the solution for notched tubes}

In this section, we shall compare the predictions of the solution with FEM results along the notch ligament. If not mentioned, all the results presented below are done for a notch geometry with an opening angle $2 \alpha$ of $60^{\circ}$ and a notch root radius $\rho$ of $1 \mathrm{~mm}$.

For accuracy purpose, a study of the mesh size has been performed for tension and bending loading using the ABAQUS commercial software [1]. For the tension case, 2D axi-symmetric model has been used with linear four noded elements 
CAX4R and for the bending case, 3D axi-symmetric model has been used with linear eight-node elements C3D8R. Both models have been set up to obtain a regular and smooth mesh in the neighborhood of the notch tip.

The geometry of the mesh as well as the results are depicted in Fig. 4. This figure presents the value of the axial concentration factor $K_{t}$ with respect to the mesh size ratio $\frac{\epsilon}{\rho}$. To reach a comfortable accuracy, the mesh size ratio has been chosen equal to 0.01 for tension loading and equal to 0.02 for bending loading. This choice is also motivated by the limitation of the number of degrees of freedom created; about 3000 and 10000 elements respectively for tension and bending loading.

Figs. 5 and 6 illustrate the distribution of the stress components $\Sigma_{Z Z}, \Sigma_{R R}$ and $\Sigma_{\Theta \Theta}$ along the notch ligament for the case of an external and internal notch under tension and bending loadings. A good agreement is found between the FEM results (markers) and the closed-form solution (line) for the axial stress component $\Sigma_{Z Z}$. Concerning the radial stress component $\Sigma_{R R}$, the semi-analytical solution is more accurate in the neighborhood of the notch tip and becomes less accurate for $r>\rho$; this error is principally due to the choice of the linear function $h(r)$ in (18). This mismatch influences slightly the distribution of the hoop stress component $\Sigma_{\Theta \Theta}$ except for the case of an internal notched tube under bending (see Fig. 6).

A way to correct this mismatch is to choose another function $h(r)$. Instead of the initial linear function introduced in (14), we propose the following hyperbolic function

$$
h(r)=\frac{\tanh \left(\frac{r-r_{o}}{\rho}\right)}{\tanh \left(\frac{l}{\rho}\right)} .
$$

However, let us remark that the form has been chosen without any a priori information or optimization, and therefore we expect that better functions can be found for this role.

In this case, an improvement is attained on the accuracy of the hoop stress $\Sigma_{\Theta \Theta}$, as for example depicted in Fig. 7. This comparison with FEM results has been obtained for the same notch geometry as before in tension loading. The improvement is less impressive in the case of bending loading.

Figs. 8 and 9 illustrate, for a variety of geometrical parameters $\alpha$ and $\rho$, the distribution of the principal stress component $\Sigma_{Z Z}$ along the notch ligament for the case of an external and internal notch under tension. Again good agreements are found between the FEM results (markers) and the closed-form solution (line).

Let us now explore the limitation of the solution with respect to the dimensions of the tube and the notch. This discussion is important in the case of a tubular 
geometry when the ligament length $l$ is of the same order of magnitude as the radius of the notch $\rho$. Indeed, the solution derived in the last sections has been obtained under some assumptions with respect to the dimensions of the tube [16], therefore the closed-form solution expressed here will not necessarily be suitable for any type of geometry. This section is therefore devoted to define the range of application of the solution.

As mentioned earlier, the parameter $m$ will take a value between 0 and $+\infty$ for a given set of notch geometry $(\rho, \alpha$ and $p)$ and tube dimension $\left(R_{i}\right.$ and $\left.R_{e}\right)$ and therefore a given stress concentration factor $K_{t}$. On this range, the axial stress concentration factor $K_{Z Z}$ defined by (25) for tension loading and (35) for bending loading is continuously decreasing, leading to a maximum value for $m=0$ and a minimum value for $m=+\infty$.

For this two limits, the function $f(r)$ introduced in (4) takes the following expressions

$$
\begin{aligned}
& \lim _{m \rightarrow 0} f(r)=\frac{r}{r_{o}}, \\
& \lim _{m \rightarrow+\infty} f(r)=1 .
\end{aligned}
$$

Introducing this two limiting functions into (25), one can derive analytically the two bounds of the axial stress concentration factor $K_{Z Z}^{t}$ in the case of tension loading

$$
\begin{aligned}
K_{Z Z}^{t, \max } & =\frac{\left(R_{e}^{2}-R_{i}^{2}\right)\left[4(q-1)+q \omega_{1}\right]}{2\left(A_{o}+B\right)}, \\
K_{Z Z}^{t, \min } & =\frac{\left(R_{e}^{2}-R_{i}^{2}\right)\left[4(q-1)+q \omega_{1}\right]}{2\left(A_{\infty}+B\right)}
\end{aligned}
$$

where the upper fix $\max$ and $\min$ are respectively related to $m=0$ and $m=\infty$. The coefficient $B$ is given by (27) and the coefficients $A_{o}$ and $A_{\infty}$ are given by

$$
\begin{aligned}
& A_{o}=4(q-1)\left[r_{o}\left(R_{o} \pm r_{o}\right) \frac{\left(1+\frac{l}{r_{o}}\right)^{\lambda_{1}}-1}{\lambda_{1}} \pm r_{o}^{2} \frac{\left.\left(1+\frac{l}{r_{o}}\right)^{\lambda_{1}+1}-1\right]}{\lambda_{1}+1}\right], \\
& A_{\infty}=4(q-1)\left\{\left(R_{o} \pm r_{o}\right) l \pm \frac{r_{o}^{2}}{2}\left[\left(1+\frac{l}{r_{o}}\right)^{2}-1\right]\right\} .
\end{aligned}
$$


For the case of bending loading, the bounds for the axial stress concentration factor $K_{Z Z}^{f}$ are obtained numerically

$$
\begin{aligned}
K_{Z Z}^{f, \max } & =\left(\frac{I}{4 R_{e}}-G\right) \frac{4(q-1)+q \omega_{1}}{C_{o}} \\
K_{Z Z}^{f, \min } & =\left(\frac{I}{4 R_{e}}-G\right) \frac{4(q-1)+q \omega_{1}}{C_{\infty}}
\end{aligned}
$$

where the coefficients $G, C_{\infty}$ and $C_{o}$ are given respectively by (36) and (37).

In order to define the range of application of the solution, we define a parameter $\xi$ characterizing the geometry of the tube which is equal to

$$
\xi=\frac{R_{i}}{R_{e}} \times \frac{p}{e}
$$

where $p$ is the depth of the notch defined by (1) and $e$ is the thickness of the tube

$$
e=R_{e}-R_{i}
$$

The parameter $\xi$ is equal to zero for a notched bar (i.e. $R_{i}=0$ ) and tends to $\infty$ for thin tubes with the tickness going to zero. We can remark that this parameter $\xi$ is not dependent on the geometry of the notch as the notch root radius $\rho$ and the half opening angle $\alpha$ and therefore the following results shall only be applied to the notch geometry tested $\left(\rho=1 \mathrm{~mm}\right.$ and $\left.\alpha=30^{\circ}\right)$.

Thereby to define the range of application of the closed-form solution, we have to find the value of the parameter $\xi$ at which the maximum axial stress concentration factor $K_{Z Z}^{\max }$ becomes smaller than the real axial stress concentration (computed with FEM).

Figs. 10 and 11 illustrate the range of validity of the closed-form solution with respect to $\xi$. These results show the evolution of the axial stress concentration factor $K_{t}$ computed with FEM solution (marked dashed lines) and the maximum axial stress concentration factor $K_{Z Z}^{\max }$ (solid lines) obtained by (42) for tension loading and (46) for bending loading. These results are obtained by varying the parameter $\xi$ for tubes presenting an internal and external notch submitted to tension and bending loading and for three different notch depths $p(1 \mathrm{~mm}, 2 \mathrm{~mm}$ and $5 \mathrm{~mm})$. To vary the parameter $\xi$, all dimensions have been kept constant excepted the internal radius $R_{i}$ for an external notch and the external radius $R_{e}$ for an internal notch. 
The figures display the intersections between $K_{t}$ and $K_{Z Z}^{\max }$ represented with a bigger marker, and one remark that all the intersection lie on a small interval $[0.21,0.27]$. Therefore we conclude that the closed-form solution is only valid for a notched tubes with a parameter $\xi$ smaller than a critical value $\xi^{*}$. The fact that $\xi$ higher than the critical value $\xi^{*}$ express the fact that the notch depth becomes large with respect to the tube thickness. This can be interpreted by saying that the solution in [8] provided in equations (4) and (5) can not be applied for this configuration, as the free internal surface of the tube is too close to the notch tip.

Moreover, as mentioned before this critical value $\xi^{*}$ should depend on the notch geometry and we have found that for $\rho=1 \mathrm{~mm}$ and $\alpha=30^{\circ}$, the critical value is equal to 0.086 .

An alternative to the numerical procedure to determine the parameter $m$ has been developed based on an empirically function depending of the notch root radius for notched bar geometry. Investigation has been performed to determine the influence of the notch root radius $\rho$, the half-opening angle $\alpha$ and the parameter $\xi$ on the parameter $m$ for notched tube geometry. The results are presented in Figs. 12 and 13 for which the computations have been done with $\xi$ equal to 0.1 . We remark in Fig. 12.a that the notch root radius $\rho$ does not influences the parameter $m$ both for an external and an internal notched tube, but has a greater influence on the opening angle $\alpha$ and the parameter $\xi$.

\section{Conclusions}

We proposed a semi-analytical stress solution suitable for notched axi-symmetrically tube submitted to tension and bending loading. This closed-form solution is a generalization of the stress solution [8] devoted to notched axi-symmetric bar. The proposed semi-analytical solution is based on an approximate elastic stress solution where the local balance condition is always satisfied by the use of a biharmonic stress function but boundary conditions are not satisfied except at specific points.

The solution has been adapted to tubular geometries by adding a supplementary term in the expression of the radial stress components $\Sigma_{R R}$. The free stress boundary condition at the inner or the outer radius could therefore be respected for externally or internally notched tubes.

The proposed semi-analytical solution has shown good agreements with respect to finite element solution in the case of an external notched tube. The match is less accurate with an internal notch on the radial and the circumferential stress components. This error has been reduced by changing the linear term in the expression of the radial stress component with a hyperbolic function as example. 
Finally a study of the validity of the semi-analytical solution regarding to the dimension of the notched tube has been performed. This study has highlighted the fact that the solution is not capable to model too high stress concentration factors. A numerical study has shown that it is possible to define a parameter $\xi$ which characterizes the geometry of the notched tube and the solution is applicable for only notched tube presenting a $\xi$ parameter smaller than $\xi^{*}$ which can be considered as independent on the notch depth but only on the notch opening angle and the notch root radius.

Moreover we have shown that the $m$ parameter introduced in (6) is highly dependent on the half opening angle $\alpha$ and the $\xi$ parameter and slightly dependent on the notch root radius $\rho$.

The application of this solution for the design of threaded components is presented in a companion paper [7].

\section{References}

[1] Abaqus 6.4. A Finite Element Commercial Software, http://www.simulia.com.

[2] B. Atzori, S. Filippi, P. Lazzarin, and F. Berto. Stress distribution in notched structural components under pure bending and combined traction and bending. Fatigue Fract. Engng. Mater. Struct., 28:13-23, 2005.

[3] H. D. Bui and A. Oueslati. Solutions exactes de fissure d'interface sous contact frottant avec un milieu indéformable. C. R. Mecanique, 332:709-716, 2004.

[4] Shin C.S. Fatigue crack growth from stress concentrations and fatigue life predictions in notched components. pages 613-652. Elsevier Science Publishers B.V., Amsterdam, 1994.

[5] Kujawski D. Estimation of stress intensity factors for small cracks at notches. Fatigue Fract. Engng. Mater. Struct., 14:953-965, 1991.

[6] M. Ferjani. Intégration des phénomènes dynamiques dans l'analyse en fatigue des garnitures de forage (Phd Thesis) Ecole Polytechnique, Palaiseau, France, 2007.

[7] M. Ferjani, A. Constantinescu, and D. Averbuch. A computational approach for the fatigue design of threaded connections Submitted to Int. J. Fatigue, 2010

[8] S. Filippi and P. Lazzarin. Distributions of the elastic principal stress due to notches in finite size plates and rounded bars uniaxially loaded. Int. J. Fatigue, 26(377-391), 2004.

[9] S. Filippi, P. Lazzarin, and R. Tovo. Developments of some explicit formulas useful to describe elastic stress fields ahead of notches in plate. Int. J. Solids \& Structures, 39:4543-4565, 2002. 
[10] N. E. Frost. Non-propagating cracks in vee-notched specimens subject to fatigue loading. The Aeronautical Quarterly, 8:1-20, 1957.

[11] G. Glinka and A. Newport. Universal feature of elastic notch-tip stress field. Int. J. Fatigue, 9(3):143-150, 1987.

[12] F. Hofmann, G. Bertolino, A. Constantinescu, and M. Ferjani. A multiscale discussion of fatigue and shakedown for notched structures. Theoretical and Applied Fracture Mechanics, 48(2):140-151, 2007.

[13] Hofmann, F., Bertolino, G., Constantinescu, A., and Ferjani, M. A discussion at the mesoscopic scale of the stress-gradient effects in high cycle fatigue based on the dang van criterion. Journal of the Mechanics of Materials and Structures, 4(2):293-308, 2009.

[14] J. Jäger. Half-planes without coupling under contact loading. ASME J. Appl. Mech., 67:247-259, 1997.

[15] Tanaka K. Engineering formulae for fatigue strength reduction due to crack-like notches. Int. J. Fracture, 22:R39-R45, 1983.

[16] P. Lazzarin and R. Tovo. A unified approach to the evaluation of linear elastic stress field in the neighborhood of cracks and notches. Int. J. Fracture, 78:3-19, 1996.

[17] Creager M. and Paris PC. Elastic field equations for blunt cracks with reference to stress corrosion cracking. Int. J. Fracture Mechanics, 3:247-252, 1967.

[18] A. S. Milyayev. The action of a concentrated force inside an elastic half-plane with a step. J. Appl. Math. Mech., 66:93-100, 2002.

[19] Williams M.L. Stress singularities resulting from various boundary conditions in angular corners of plates in tension. ASME J. Appl. Mech., 19:526-528, 1952.

[20] F. Morel and T. Palin-Luc. A non-local theory applied to high cycle multiaxial fatigue. Fatigue Fract. Engng. Mater. Struct., 25:649-665, 2002.

[21] N.I. Mushkelishvili. Some Basics Problems of the Mathematical Theory of Elasticity. Noordhoof Leyden, 1953.

[22] H. Neuber. Theory of notch stresses: Principles for exact stress calculation. Berlin, 1958. Springer-Verlag. Translation of Kerbspannungslehre : Grundlagen Fuer Genaue Spannunggrechnung.

[23] Lazzarin P., Tovo R., and Meneghetti G. Fatigue crack initiation and propagation phases near notch in metals with low notch sensitivity. Int. J. Fatigue, 19(8-9):647657, 1997.

[24] I. V. Papadopoulos and V. P. Panoskaltsis. Invariant formulation of a gradient dependent multiaxial high-cycle fatigue criterion. Engng. Fract. Mech., 55(4):513$528,1996$.

[25] G. Pluvinage. Fatigue and fracture emanating from notch; the use of the notch stress intensity factor. Nuclear Engng Design, 185:173-184, 1998. 
[26] D.L. Du Quesnay, M.T. Yu, and T.H. Topper. An analysis of notch-size effects at the fatigue limit. J. Testing \& Mat, 16(4):375-385, July 1988.

[27] Xu RX., Thompson J.C., and Topper T.H. Practical stress expressions for stress concentration regions. Fatigue Fract. Engng. Mater. Struct., 18:885-895, 1995.

[28] G. N. Savin. Stress Concentration Around Holes. Pergamon Press, New York, 1961.

[29] J. Schijve. Stress gradients around notches. Fatigue Engng. Mater. Struct., 5:325-332, 1982.

[30] D. Taylor. Geometrical effects in fatigue: A unified theoretical model. Int. J. Fatigue, 21:413-420, 1999.

[31] M.L. Williams. On the stress distribution at the base of a stationary crack. ASME J. Appl. Mech., 24:109-114, 1957.

\section{Appendix 1}

Recently, a set of closed-form equations valid for V-shaped notched axi-symmetric bar under Modes I and II loading has been proposed in [8]. The stress field has been obtained by the use of the Kolosoff-Mushkelishvili's complex potential function [21] and the following conformal mapping introduced by Neuber [22]

$$
z=x+i y=(u+i v)^{q}=\xi^{q}
$$

where $z$ is the complex representation of the cartesian coordinates $\{x, y\}$ and $\{u, v\}$ is the auxiliary system of curvilinear coordinates (see Fig. 14).

The parameter $q$ in (50) is linked to the opening angle $2 \alpha$ by the following relation

$$
q=2\left(1-\frac{\alpha}{\pi}\right)
$$

The origin of the cartesian coordinate system is located at a distance $r_{o}$ from the tip of the notch, where $r_{o}$ depends on the notch root radius $\rho$ and the opening angle $2 \alpha$ according to

$$
r_{o}=\rho \frac{q-1}{q}=\rho \frac{\pi-2 \alpha}{2 \pi-2 \alpha} .
$$

For Mode I loading, the stress field along the notch bisector, i.e. $\theta=0$, is 


$$
\begin{gathered}
\left.\sigma_{\theta}\right|_{\theta=0}=\sigma_{y}=\frac{\sigma_{\max }}{4(q-1)+q \omega_{1}}[4(q-1)\{1 \\
\left.+\frac{\operatorname{atan}\left[m\left(r-r_{o}\right)\right]}{m r_{o}}\right\}^{\lambda_{1}-1} \\
\left.+q \omega_{1}\left(\frac{r}{r_{o}}\right)^{\mu_{1}-1}\right] \\
\left.\sigma_{r}\right|_{\theta=0}=\sigma_{x}=\frac{\sigma_{\max }}{\eta_{1}\left[4(q-1)+q \omega_{1}\right]}\left[4(q-1)\left(\frac{r}{r_{o}}\right)^{\lambda_{1}-1}+q \delta_{1}\left(\frac{r}{r_{o}}\right)^{\mu_{1}-1}\right]
\end{gathered}
$$

where the parameters introduced in (53) and (54) are equal to

$$
\begin{aligned}
\omega_{1} & =\frac{\chi_{d_{1}}\left(1+\mu_{1}\right)+\chi_{c_{1}}}{1+\lambda_{1}+\chi_{b_{1}}\left(1-\lambda_{1}\right)}, \\
\eta_{1} & =\frac{1+\lambda_{1}+\chi_{b_{1}}\left(1-\lambda_{1}\right)}{3-\lambda_{1}-\chi_{b_{1}}\left(1-\lambda_{1}\right)}, \\
\delta_{1} & =\frac{\chi_{d_{1}}\left(3-\mu_{1}\right)-\chi_{c_{1}}}{3-\lambda_{1}-\chi_{b_{1}}\left(1-\lambda_{1}\right)}, \\
\chi_{b_{1}} & =\frac{\sin \left[\left(1-\lambda_{1}\right) q \pi / 2\right]}{\sin \left[\left(1+\lambda_{1}\right) q \pi / 2\right]}, \\
\chi_{c_{1}} & =\left[\left(1-\mu_{1}\right)^{2}-\frac{1+\mu_{1}}{q}\right]\left[3-\lambda_{1}-\chi_{b_{1}}\left(1-\lambda_{1}\right)\right]-\left(3-\mu_{1}\right) \varepsilon_{1}, \\
\chi_{d_{1}} & =\left[\frac{1-q\left(1+\mu_{1}\right)}{q}\right]\left[3-\lambda_{1}-\chi_{b_{1}}\left(1-\lambda_{1}\right)\right]-\varepsilon_{1}, \\
\varepsilon_{1} & =\left(1-\lambda_{1}\right)^{2}+\chi_{b_{1}}\left(1-\lambda_{1}^{2}\right)-\frac{1+\lambda_{1}-\chi_{b_{1}}\left(1-\lambda_{1}\right)}{q}
\end{aligned}
$$

and the constants $\lambda_{1}$ and $\mu_{1}$ are such that they satisfy the following equations

$$
\begin{aligned}
& \sin \left(\lambda_{1} q \pi\right)+\lambda_{1} \sin (q \pi)=0, \\
& \left\{\frac{1-q\left(1+\mu_{1}\right)}{q}\left[3-\lambda_{1}-\chi_{b_{1}}\left(1-\lambda_{1}\right)\right]-\varepsilon_{1}\right\}\left(1+\mu_{1}\right) \varepsilon_{1} \cos \left[\left(1-\mu_{1}\right) q \frac{\pi}{2}\right]+ \\
& \left\{\left[\left(1-\mu_{1}\right)^{2}-\frac{1+\mu_{1}}{q}\right]\left[3-\lambda_{1}-\chi_{b_{1}}\left(1-\lambda_{1}\right)\right]-\left(3-\mu_{1}\right) \varepsilon_{1}\right\} \\
& \times \cos \left[\left(1+\mu_{1}\right) q \frac{\pi}{2}\right]=0 .
\end{aligned}
$$

Table 1 provides useful values of the different parameters for various opening angle $2 \alpha$ 


\begin{tabular}{|l|l|l|l|l|l|l|l|l|l|}
\hline $2 \alpha\left[^{\circ}\right]$ & $q$ & $\lambda_{1}$ & $\mu_{1}$ & $\chi_{b_{1}}$ & $\chi_{c_{1}}$ & $\chi_{d_{1}}$ & $\delta_{1}$ & $\eta_{1}$ & $\omega_{1}$ \\
\hline \hline 0 & 2 & 0.5 & -0.5 & 1 & 4 & 0 & -2 & 1 & 2 \\
\hline 30 & 1.83 & 0.502 & -0.456 & 1.07 & 3.79 & 0.063 & -1.82 & 1.04 & 1.88 \\
\hline 60 & 1.67 & 0.512 & -0.406 & 1.31 & 3.28 & 0.096 & -1.6 & 1.17 & 1.55 \\
\hline 90 & 1.5 & 0.544 & -0.345 & 1.84 & 2.51 & 0.105 & -1.33 & 1.47 & 1.08 \\
\hline 120 & 1.33 & 0.616 & -0.268 & 3 & 1.51 & 0.087 & -1 & 2.25 & 0.57 \\
\hline
\end{tabular}

Table 1

Value parameters of the closed-form solution for various opening angle $2 \alpha$.

\section{Figures}



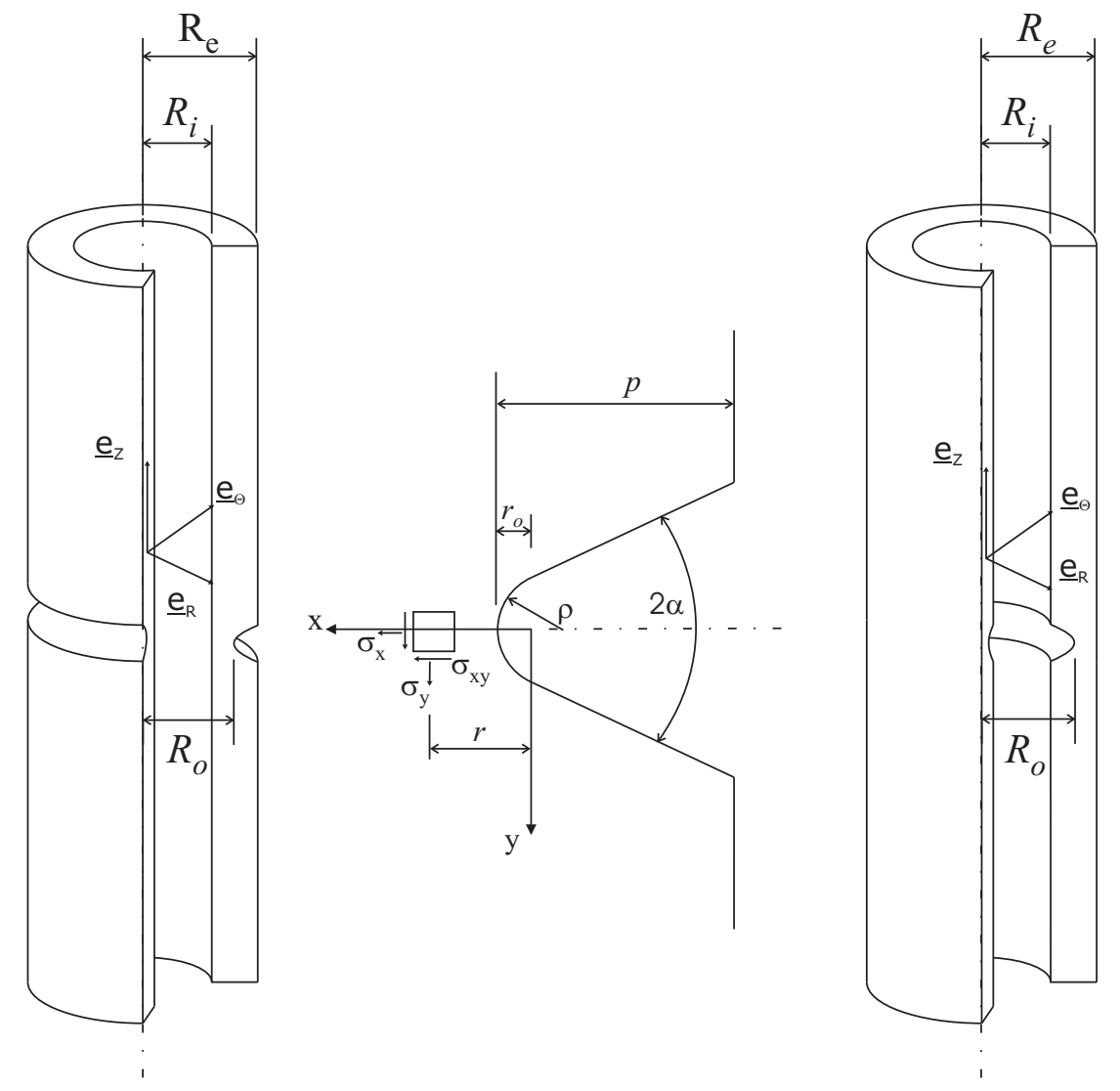

Figure 1. Geometry of tubular components with an internal (right) and external (left) notch and geometry of the notch (center). 

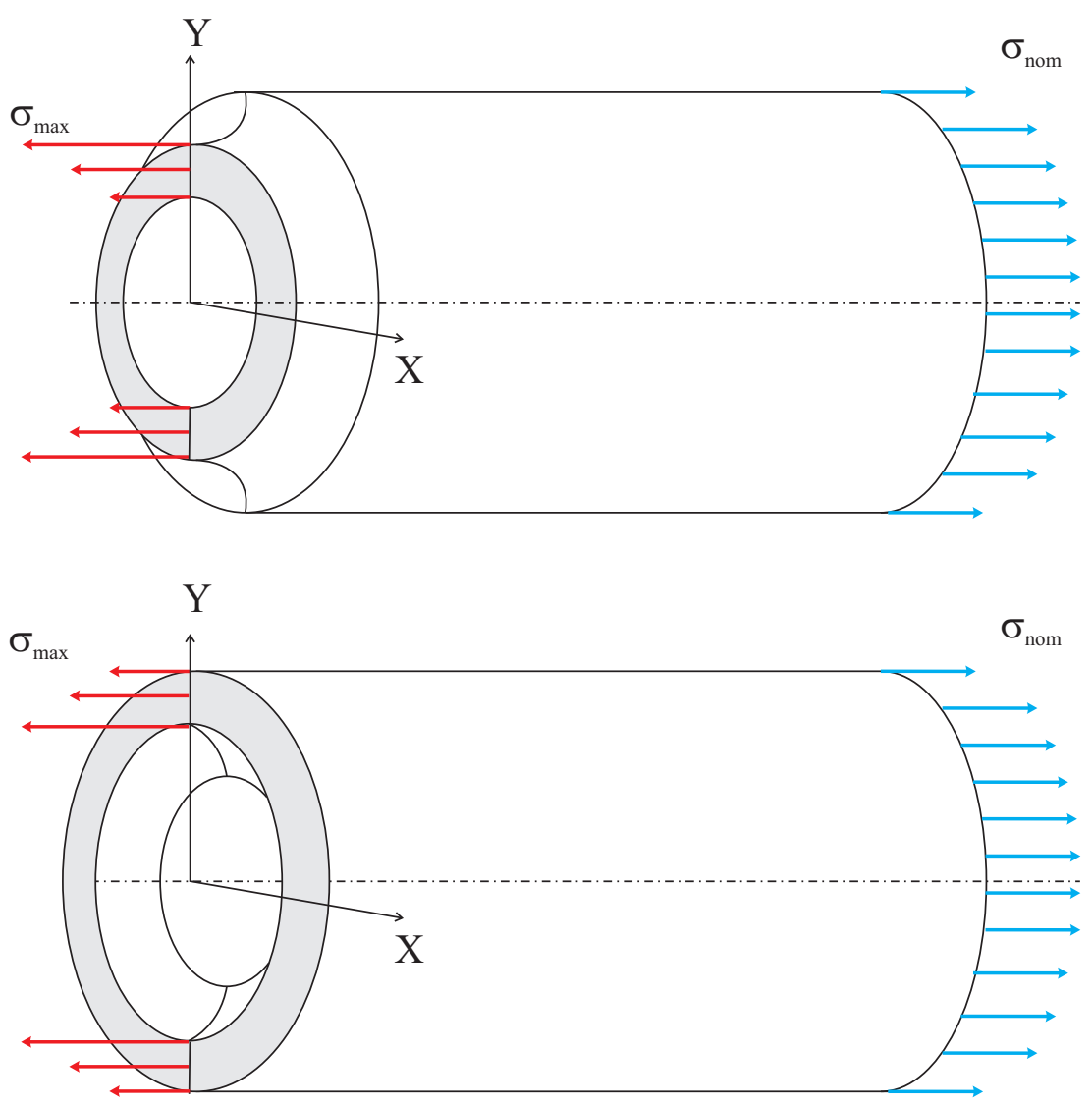

Figure 2. Schematic view of the distribution of the axial stress component $\Sigma_{Z Z}$ along the ligament for tension loading. 

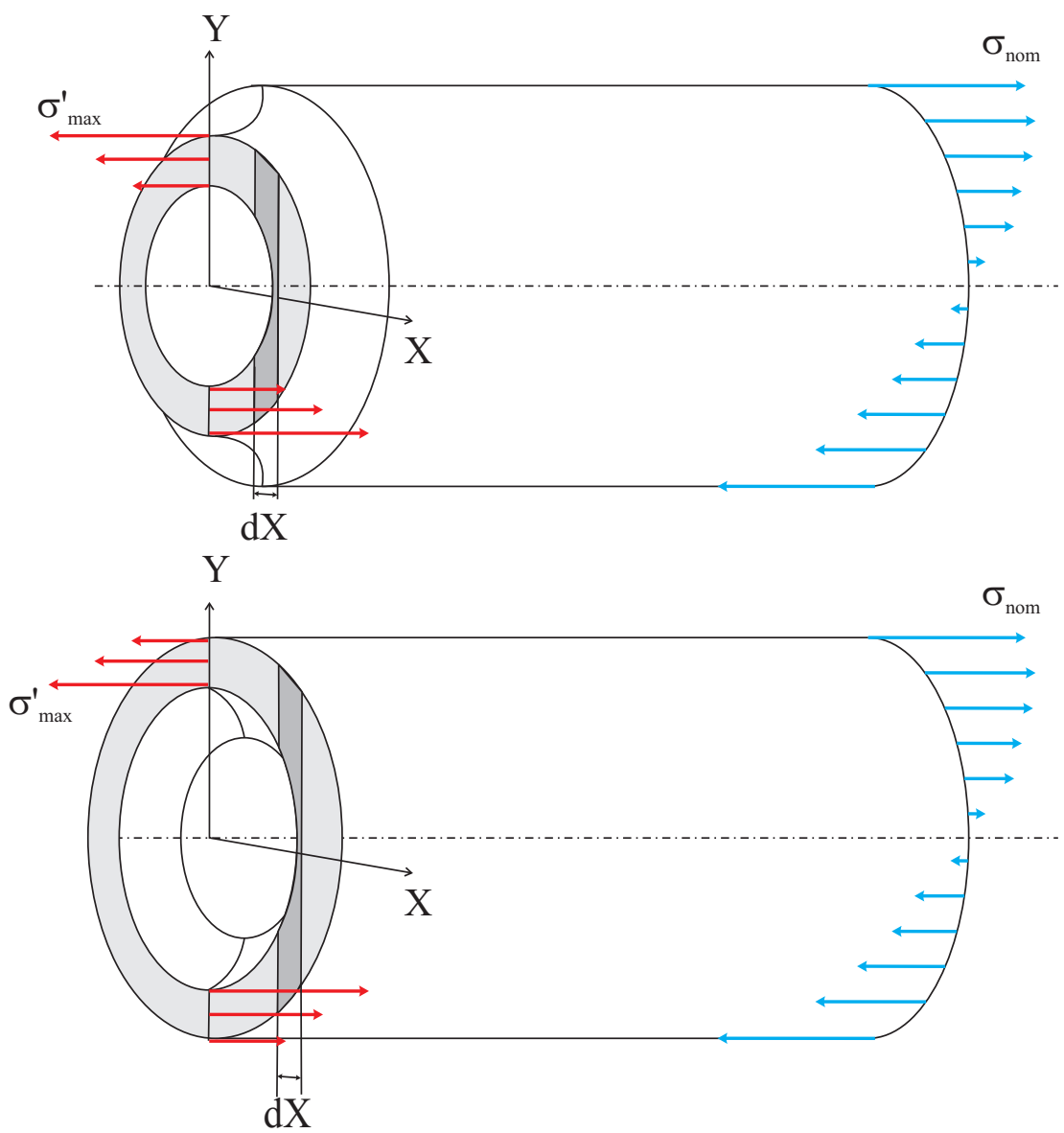

Figure 3. Schematic view of the distribution of the axial stress component $\Sigma_{Z Z}$ along the ligament for bending loading. 


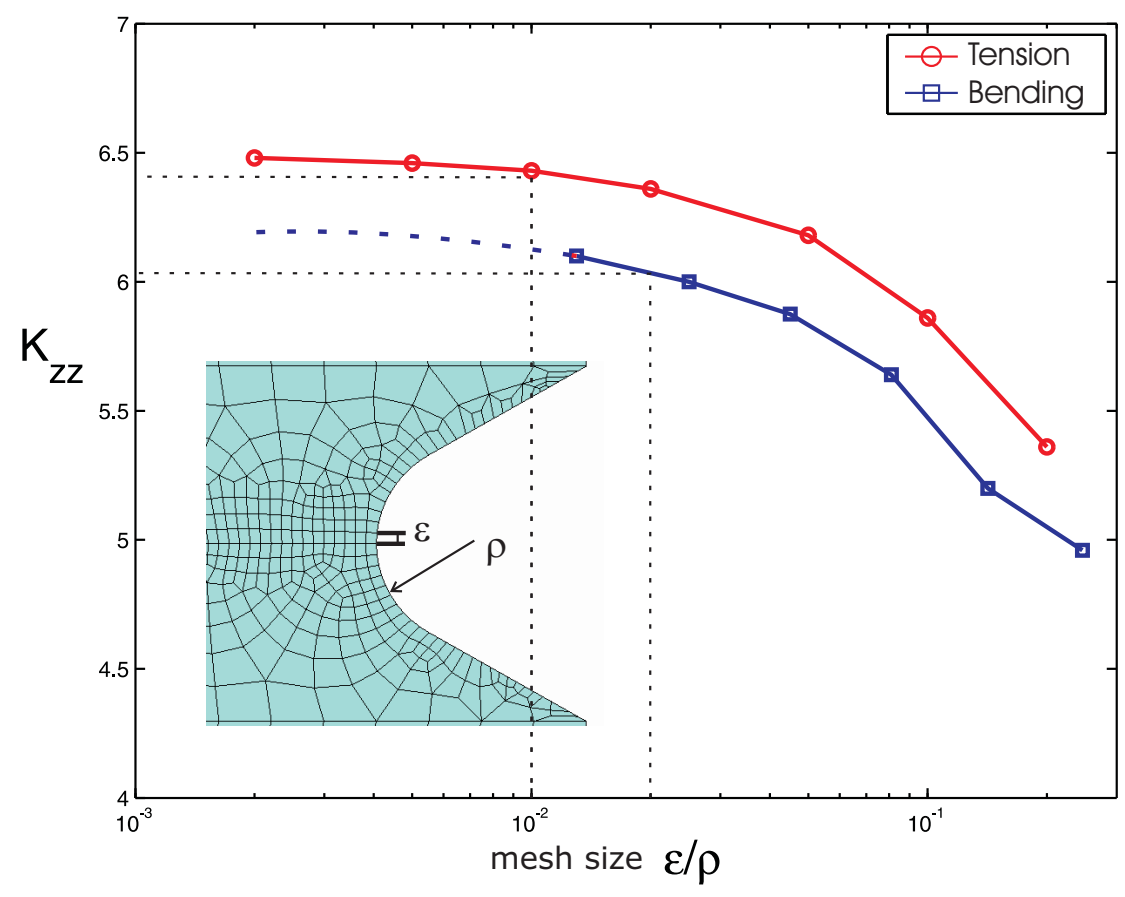

Figure 4. Mesh size sensitivity for an externally notched tube with $2 \alpha=60^{\circ}$ and $\rho=1 \mathrm{~mm}$. 


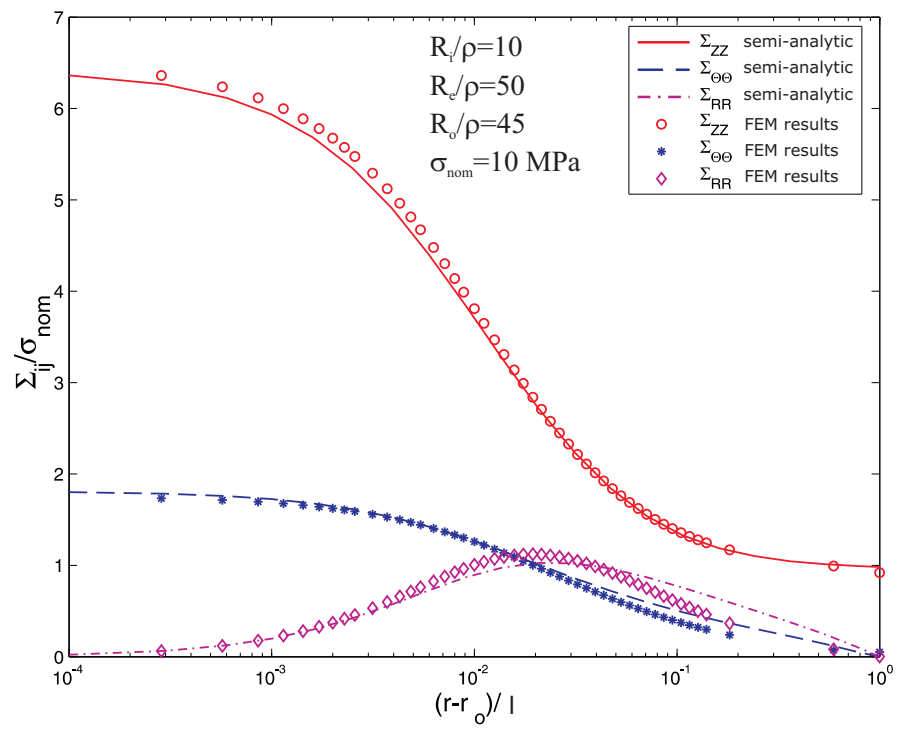

a) tension loading

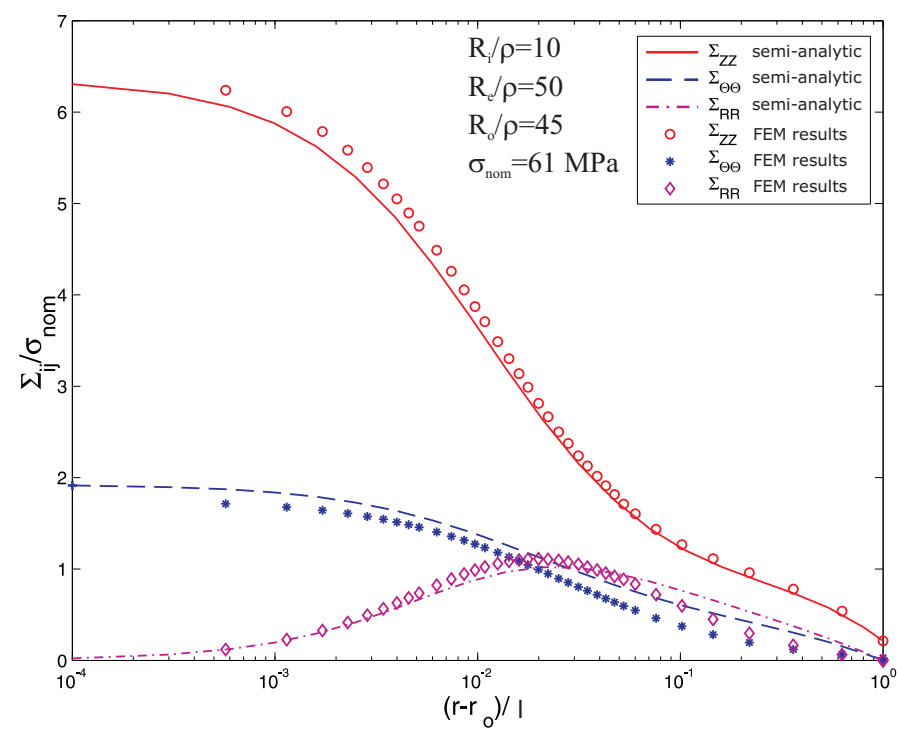

b) bending loading

Figure 5. Stress field distribution along the ligament of an external notch. 


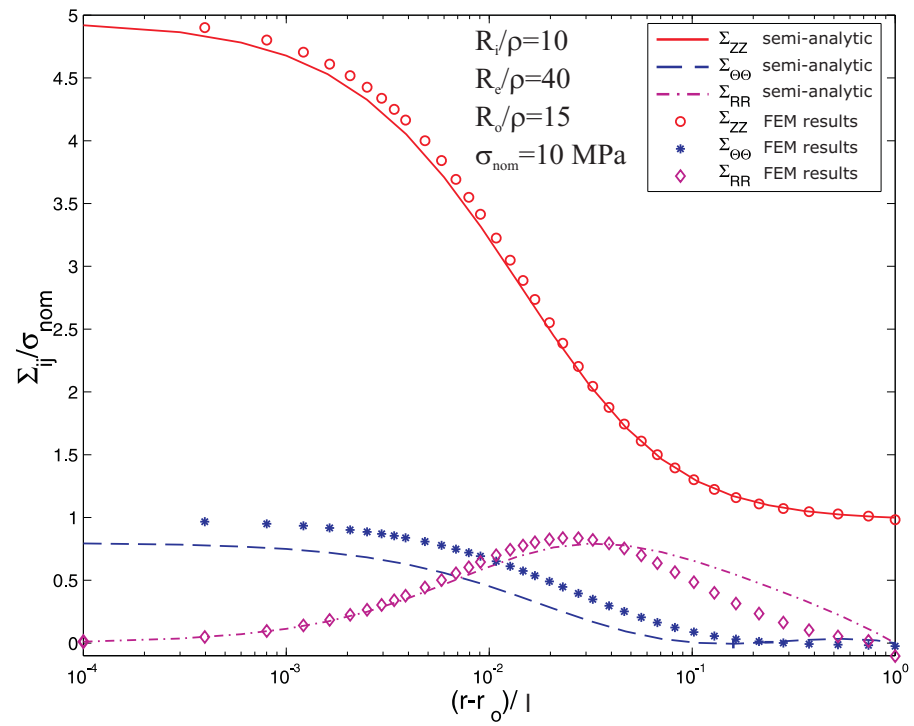

a) tension loading

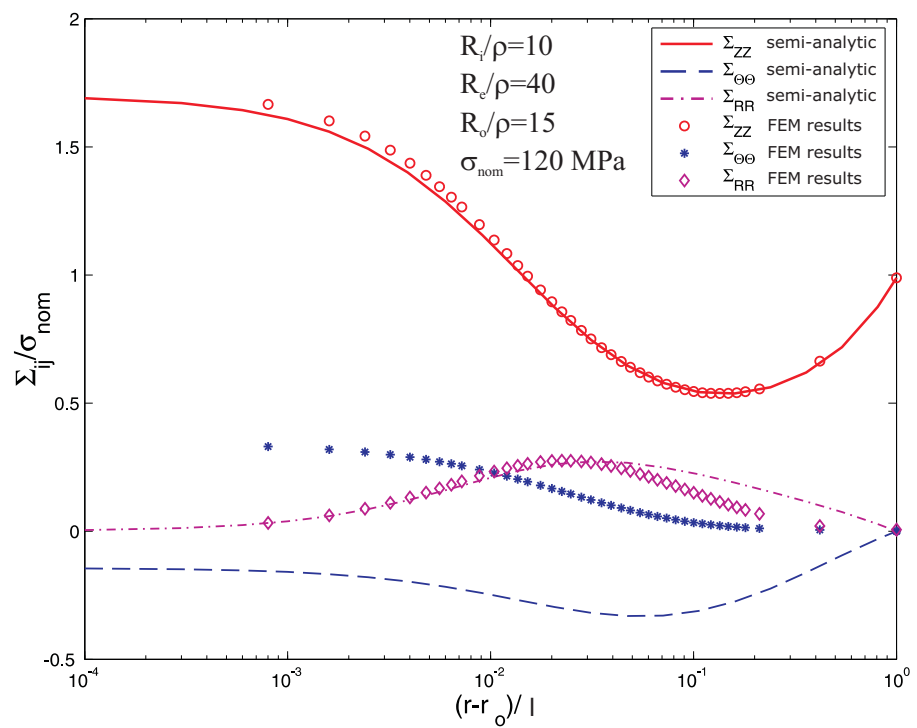

b) bending loading

Figure 6. Stress field distribution along the ligament of an internal notch. 

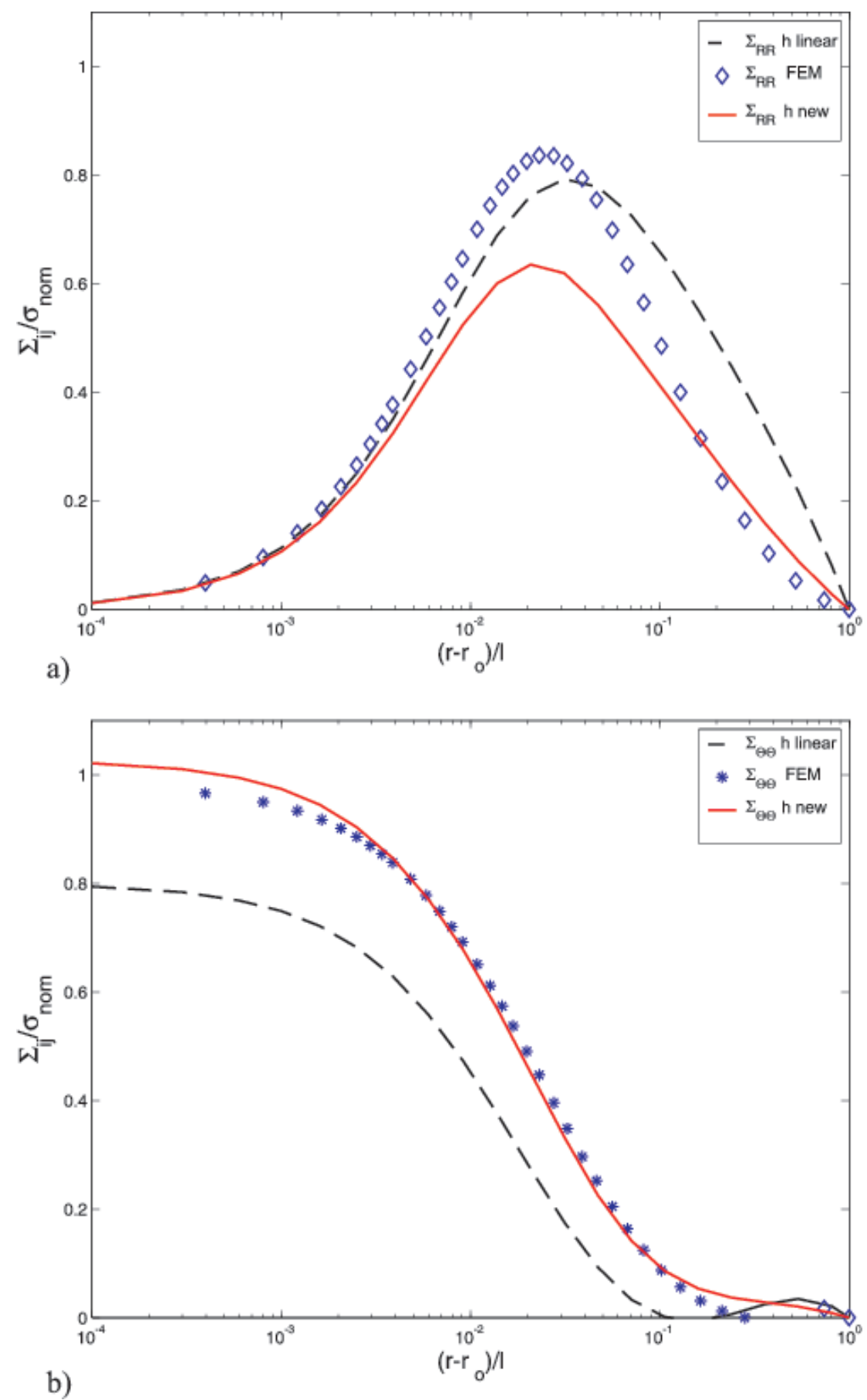

Figure 7. Distribution of the radial (a) and the hoop stress (b) components under tensile loading in the case of an internal notch for differents choices of $h$ : linear see equation (14) and the new nonlinear $h$ see equation (39). 


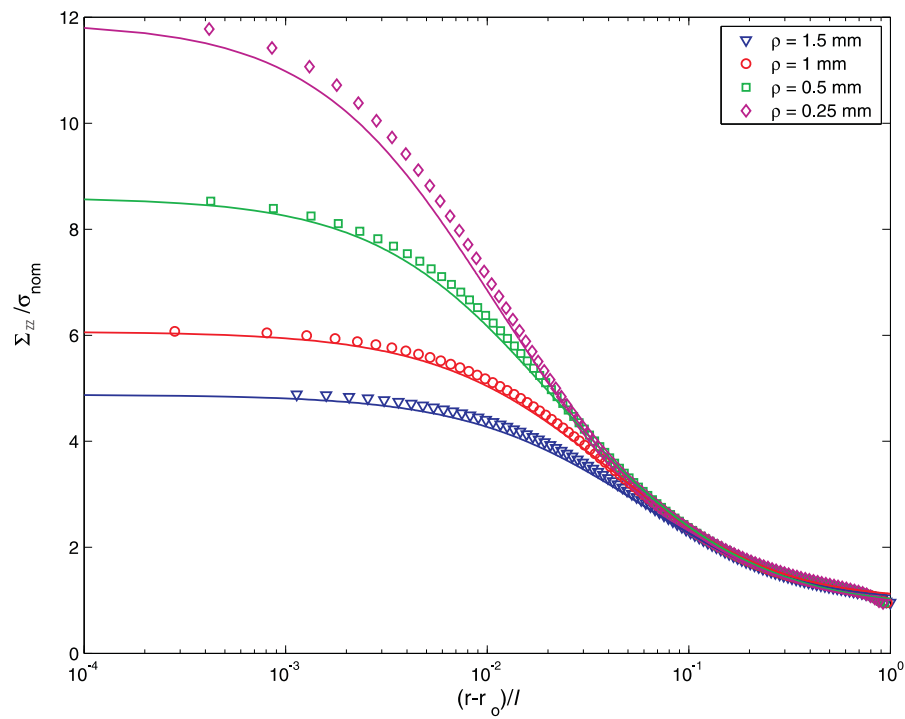

a)

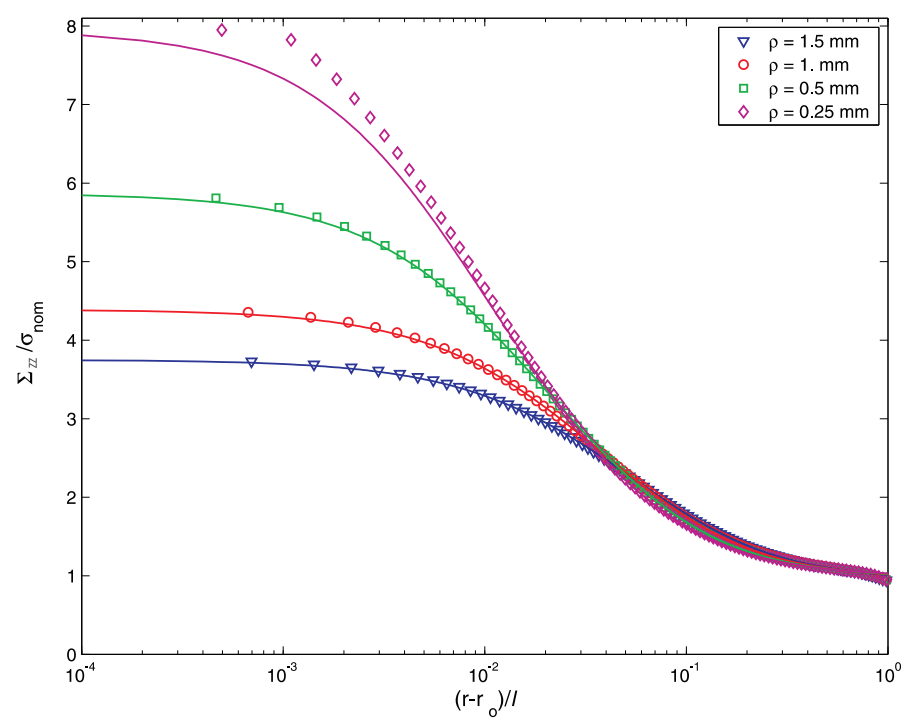

b)

Figure 8. Distribution of the axial stress component for different notch root radius under tension loading and with $\alpha=40^{\circ}$ for (a) an externally notched tube (b) an internally notched tube. 


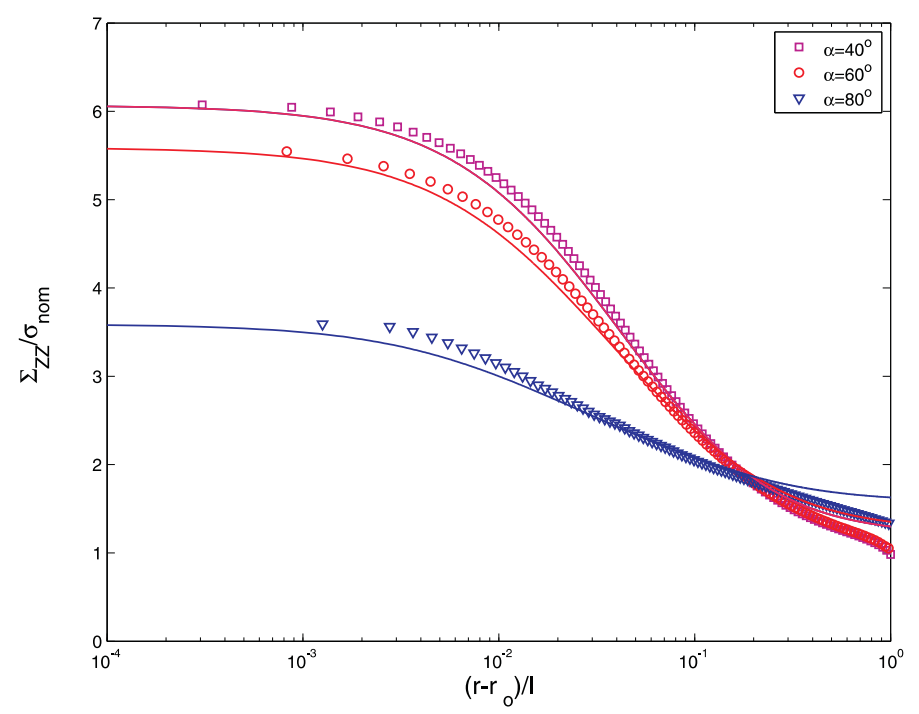

a)

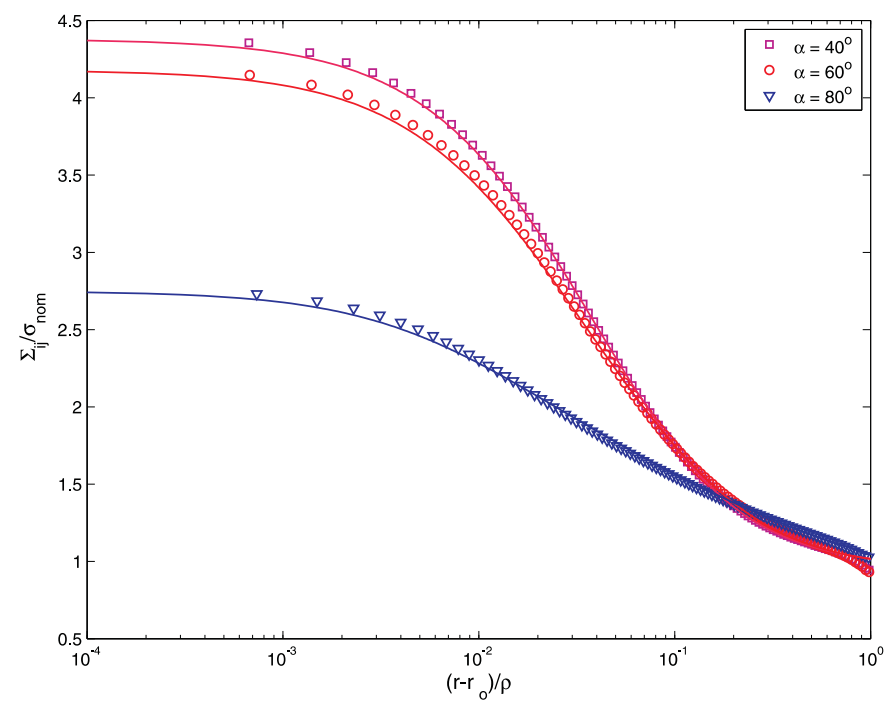

b)

Figure 9. Distribution of the axial stress component for different opening angle under tension loading and with $\rho=1 \mathrm{~mm}$ for (a) an externally notched tube (b) an internally notched tube. 


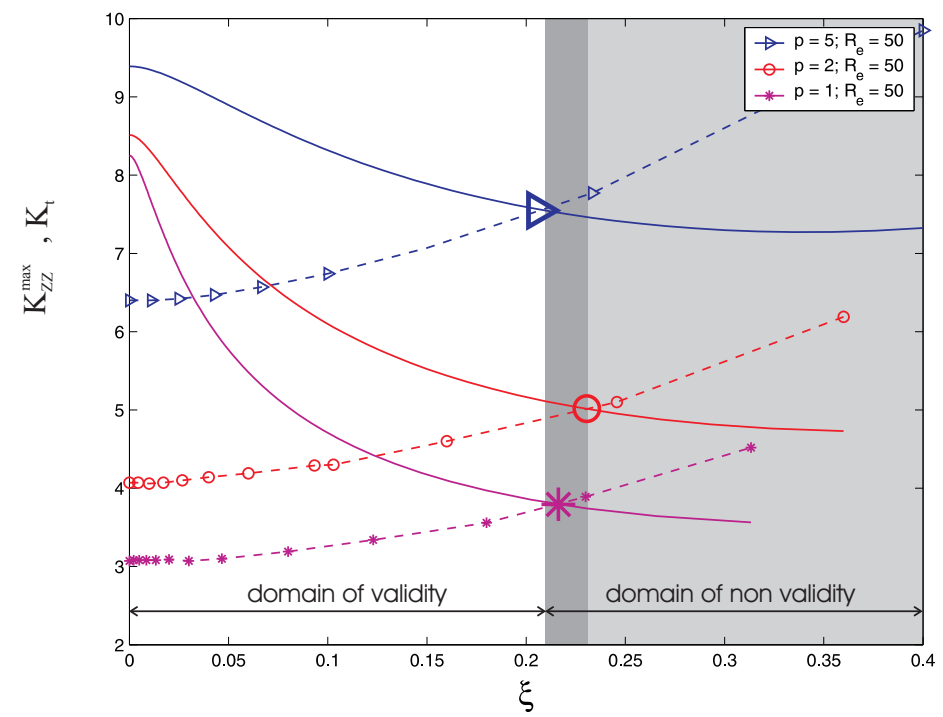

a) tension loading

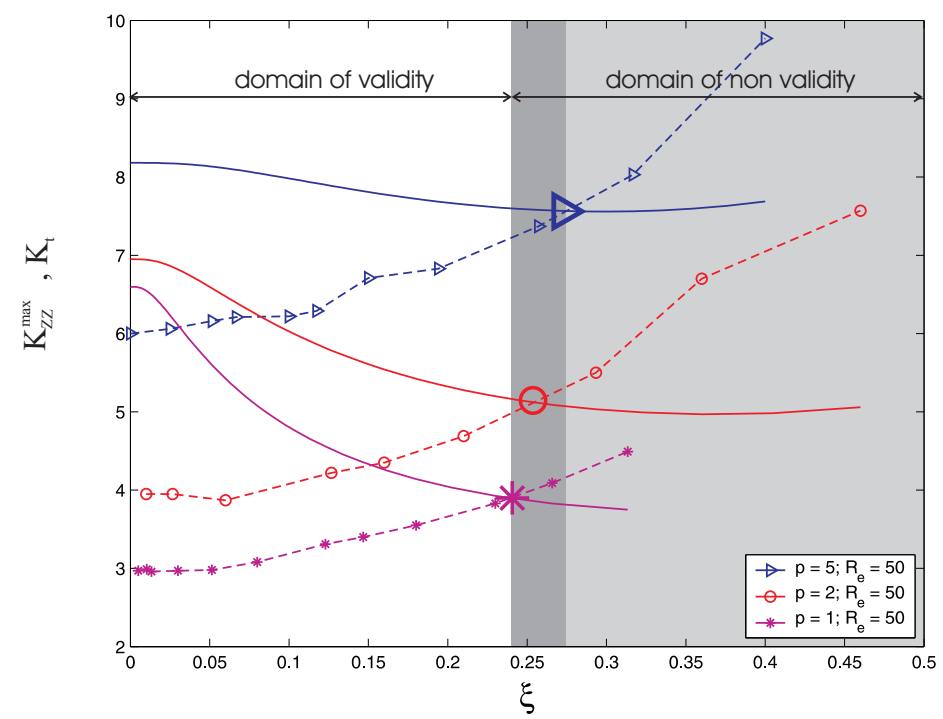

b) bending loading

Figure 10. Limiting values of the axial stress concentration factor $K_{t}$ computed by FEM (marked dashed lines) and the maximum axial stress concentration factor $K_{Z Z}^{\max }$ (solid lines) predicted with the semi-analytical solution for different tubular geometries for an external notch. 


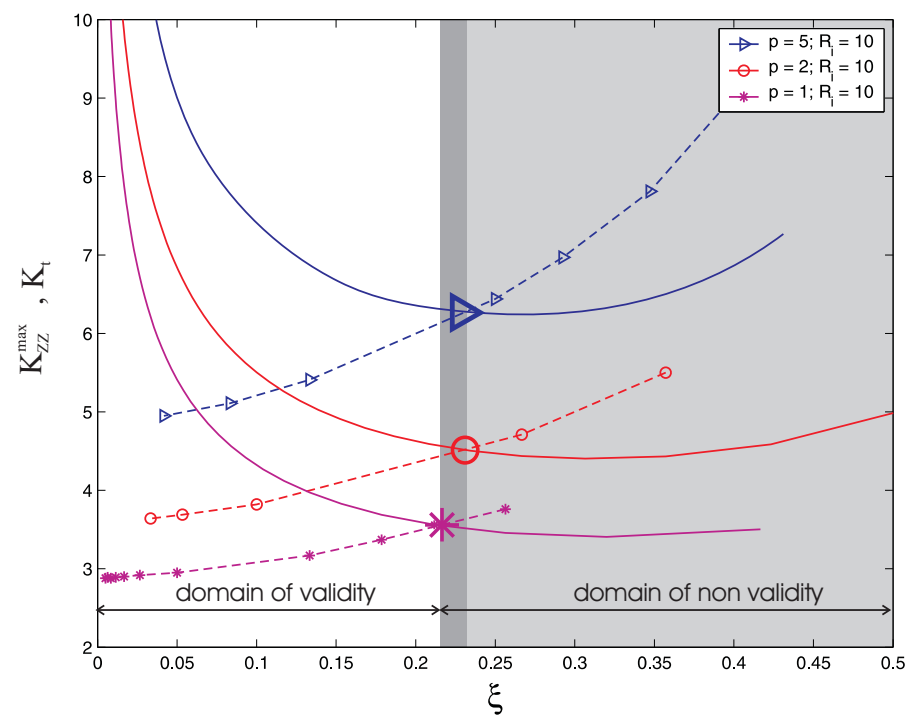

a) tension loading

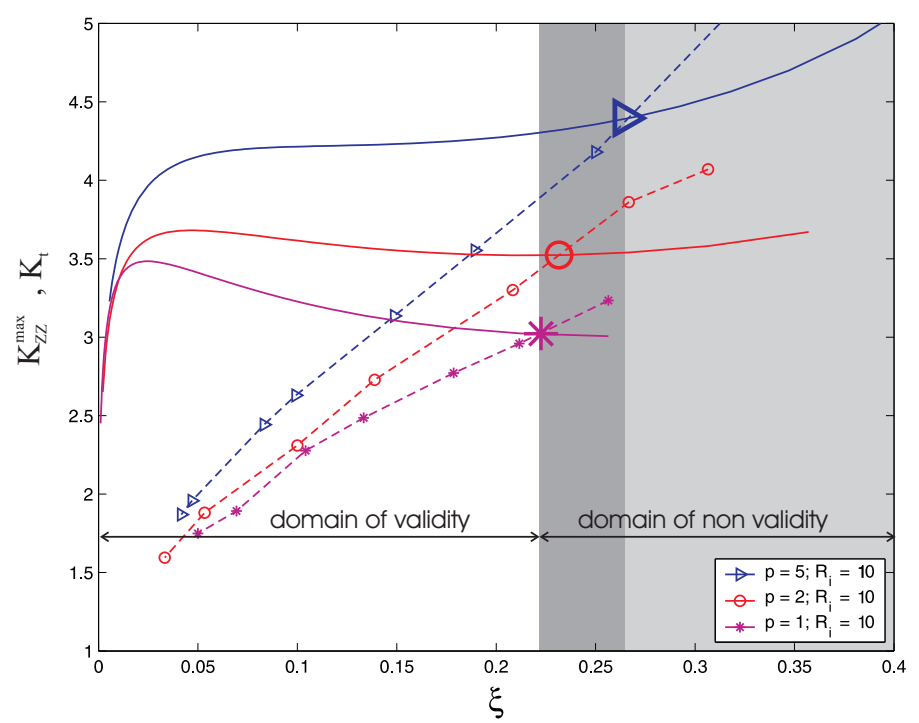

b) bending loading

Figure 11. Limiting values of the axial stress concentration factor $K_{t}$ computed by FEM (marked dashed lines) and the maximum axial stress concentration factor $K_{Z Z}^{\max }$ (solid lines) predicted with the semi-analytical solution for different tubular geometries for an internal notch. 


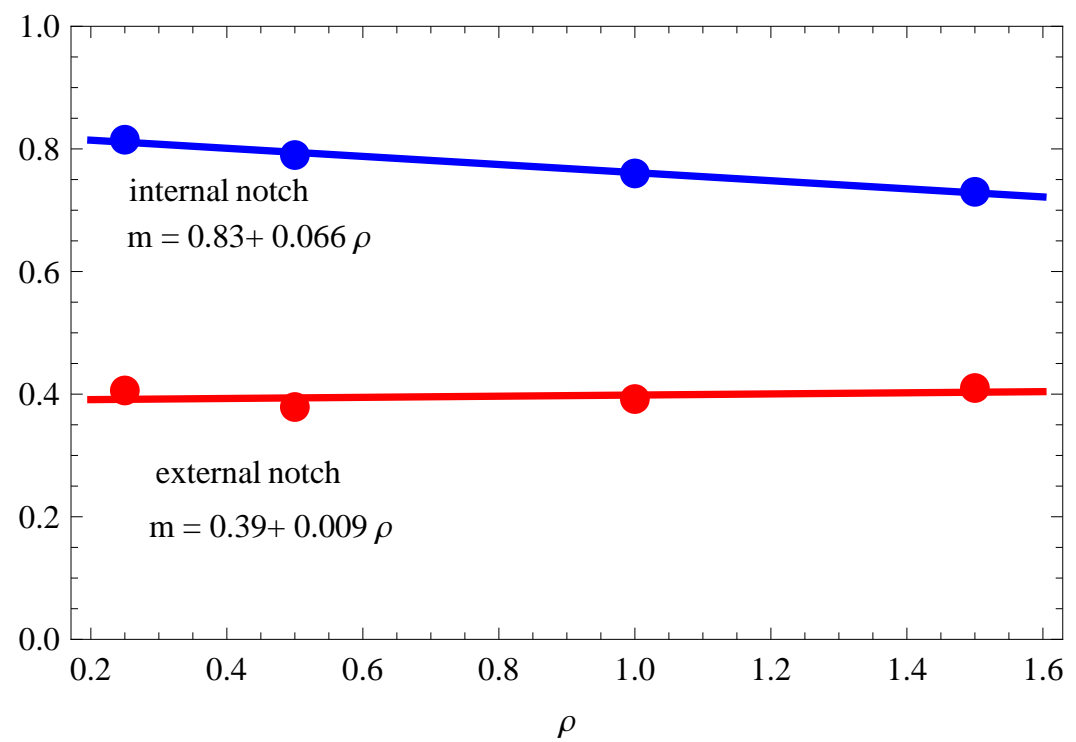

(a)

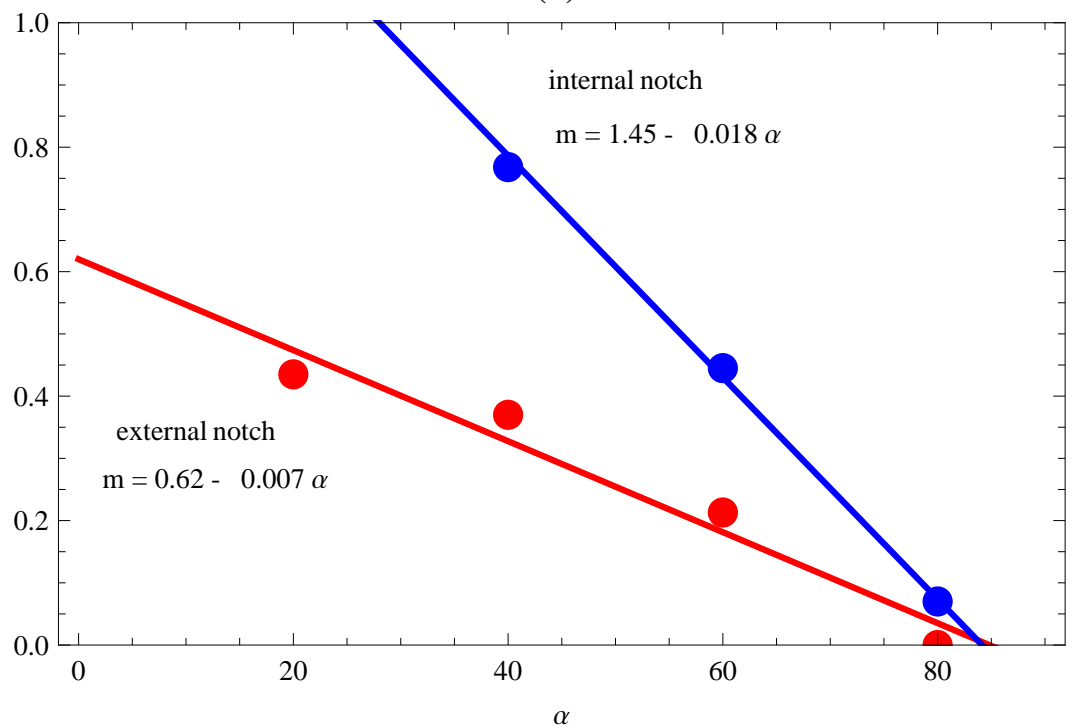

(b)

Figure 12. Evolution of the parameter $m$ (a) for various notch radius $\rho$ with $\alpha=40^{\circ}$ and (b) for various notch opening angle $\alpha$ with $\rho=1 \mathrm{~mm}$. 


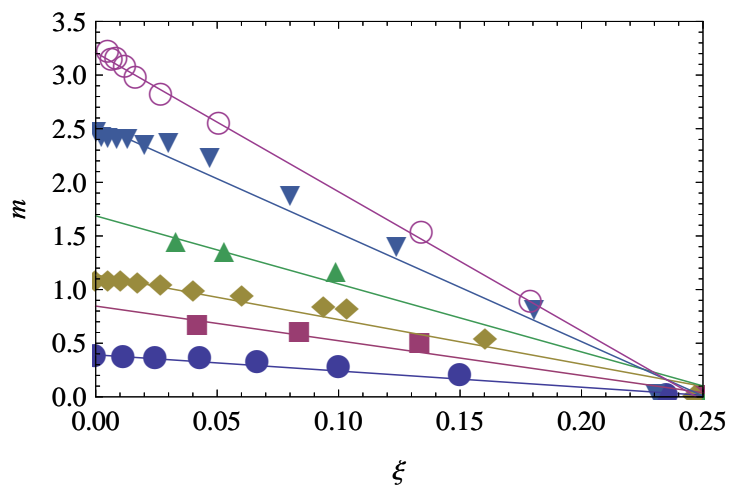

$\begin{array}{ll}\rho=5 \mathrm{~mm} \text { ext } \\ & \rho=5 \mathrm{~mm} \text { int } \\ \boldsymbol{\Delta} & \rho=2 \mathrm{~mm} \text { ext } \\ \nabla & \rho=2 \mathrm{~mm} \text { int } \\ & \rho=1 \mathrm{~mm} \text { ext } \\ \end{array}$

Figure 13. Evolution of the parameter $m$ for various $\xi$ with $\alpha=30^{\circ}$. 


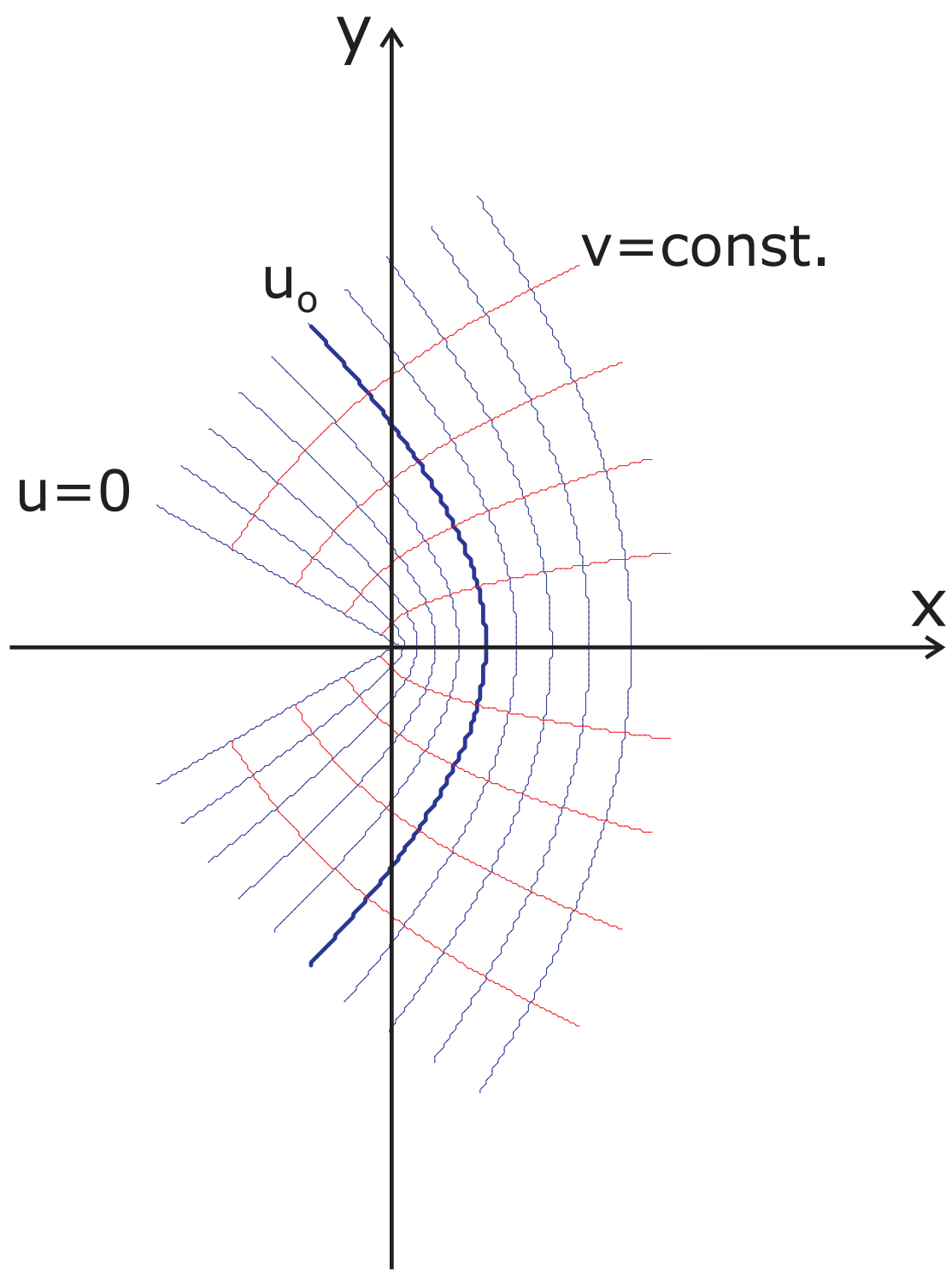

Figure 14. Representation of the auxiliary system of coordinates $\{u, v\}$. The curve $u=u_{o}$ represents the geometry of the notch. 